\title{
DEFINIÇÃO DE COMPETÊNCIAS EM CUIDADOS PALIATIVOS NA FORMAÇÃO DO MÉDICO GENERALISTA
}

\author{
Kátia Macário Santos Quintiliano¹ e Francisco José Passos Soares1
}

\author{
1Universidade Federal de Alagoas, Brasil. kalmacario@hotmail.com; francisco_passos01@hotmail.com
}

\begin{abstract}
Resumo. Introdução: A matriz curricular de uma escola médica deve concordar com a prática profissional e ser pautada pelas necessidades da sociedade. No Brasil, a formação médica apresenta deficiência na abordagem dos cuidados paliativos. Objetivo: Definir as competências em cuidados paliativos necessárias para a formação do médico generalista. Metodologia: Pesquisa descritiva e exploratória utilizando-se da técnica Delphi. A amostra foi constituída por 11 médicos onde suas especialidades permitem ter área de atuação em cuidados paliativos. Na primeira rodada de perguntas foram elencadas competências que os profissionais consideraram necessárias para o médico generalista; na segunda os participantes escolheram as competências após o compilado da primeira rodada, com o acréscimo de algumas competências citadas no Libro Blanco; e na terceira rodada foi validada a matriz de competências elaborada por consenso. Resultados: As recomendações mais votadas pelos painelistas foram: conhecimento sobre cuidados paliativos, cuidados paliativos na atenção básica, controle de sintomas, trabalho interprofissional, habilidades de comunicação e bioética. Uma matriz com 30 competências foi desenvolvida. Conclusões: A técnica Delphi é uma importante ferramenta de pesquisa qualitativa que proporcionou a elaboração de uma matriz de competências em cuidados paliativos, a qual poderá contribuir na qualificação da formação médica e na assistência à saúde da população.
\end{abstract}

Palavras-chave: Cuidados Paliativos; Medicina; Competência; Curriculo Médico.

\section{COMPETENCES ON PALLIATIVE CARE IN THE TRAINING OF GENERAL PRACTITIONERS}

\begin{abstract}
Introduction: The curricular matrix of a medical school should agree with the professional practice and based on social necessities. In Brazil, the medical undergraduate has deficiencies in the palliative cares approach. Objective: To define the necessary skills and palliative cares at the general practitioner's formation. Method: Descriptive and exploratory research using the Delphi technique. The sample was composed of 11 medical doctors where the specialty allows the practice in the palliative care field. At the first round of questions, the skills listed were that the professionals considered necessary to the general practitioner; at the second round, the participants choose the skills before the list of the first turn, with the skills cited on White Book added; the third round the skills matrix was elaborate and validated by the consensus. Results: The panellist's most voted recommendations were: palliative care knowledge, palliative care in basic attention, symptom control, interprofessional work, communication skills and bioethics. A matrix with 30 skills in palliative care was developed. Conclusions: The Delphi technique is an important qualitative research tool that provided the elaboration of skills in the palliative care matrix, that can contribute to the medical undergraduate qualification and the people's health assistance.
\end{abstract}

Keywords: Palliative Care; Medicine; Skills; Medical Curriculum.

\section{INTRODUÇÃO}

O Brasil apresenta uma mudança demográfica similar à de outros países em desenvolvimento, com redução das taxas de natalidade e mortalidade, indicando prolongamento da expectativa de vida e envelhecimento da população (Carvalho \& Garcia, 2003; Ministério da Saúde [MS], 2007).. Com o aumento da população idosa aumentam 
também as Doenças Crônicas Não Transmissíveis (DCNT), que têm respondido por cerca de $75 \%$ das mortes e perda de qualidade de vida (Souza, Malta, França, \& Barreto, 2018).

Para cuidar destes pacientes, estão sendo estudados e desenvolvidos os cuidados paliativos, que consistem na atenção promovida de forma multidisciplinar e interdisciplinar com o objetivo de melhorar a qualidade de vida do paciente e de seu familiar diante de uma doença ameaçadora da vida, com prevenção e alívio do sofrimento, tratamento impecável da dor e de outros sintomas físicos, sociais, psicológicos e espirituais (World Health Organization, 2002).

Estudos feitos em diferentes países apontam a necessidade da presença dos cuidados paliativos no currículo de Medicina, reconhecendo o envelhecimento populacional como principal justificativa e a consideração das competências em cuidados paliativos, ambos fundamentais para os médicos generalistas (Denney-Koelsch, Horowitz, Quill, \& Baldwin, 2018; Horowitz, Gramling, \& Quill, 2014; Cheng \& Teh, 2014).

Desde o ano 2000, várias instituições de ensino nos Estados Unidos incluíram no currículo médico aspectos importantes sobre o cuidado no fim da vida desde o início do curso, enquanto outras apresentam palestras ou conteúdos menos estruturados (Horowitz et al., 2014)

O Conselho das Recomendações Europeias para os Estados Membros sobre a organização dos cuidados paliativos (Council of Europe, 2003) destaca a necessidade de incorporação de programas estruturados de educação não somente na formação de médicos, mas de todos os profissionais envolvidos nos cuidados de saúde. Em 2013, a Associação Europeia em Cuidados Paliativos (EAPC) desenvolveu um documento com as competências centrais para atuar em cuidados paliativos direcionado aos médicos e outros profissionais da saúde na Europa (Associação Europeia de Cuidados Paliativos [EAPC], (2013).

Em outubro de 2018, o Ministério da Saúde do Brasil publicou a Resolução no 41 (2018), normatizando os cuidados paliativos no SUS. O documento, em seu artigo $3^{\circ}$ - que versa sobre os objetivos da organização dos cuidados paliativos no SUS -, menciona no parágrafo IV o objetivo de fomentar a instituição de disciplinas e conteúdos programáticos de cuidados paliativos no ensino de graduação e especialização dos profissionais de saúde. 
No entanto, o conhecimento profissional insuficiente para atuar na área de cuidados paliativos tem acarretado dificuldades para uma assistência adequada (Floriani \& Schramm, 2007). Apesar da literatura existente sobre bioética e cuidados paliativos, a pesquisa sobre o ensino nesta área é insuficiente e a maioria dos cursos de Medicina no Brasil ainda não adota formação orientada para os cuidados paliativos (Oliveira, Ferreira, \& Rezende 2013).

Com a insuficiência teórico-prática e metodológica no ensino sobre os cuidados paliativos nos cursos de Medicina, colocou-se a perspectiva de investigação para a definição das competências necessárias para a composição do perfil do médico generalista.

\section{METODOLOGIA}

Estudo exploratório-descritivo, realizado entre os meses de dezembro de 2018 e junho de 2019, utilizando-se da técnica de Delphi. A adoção dessa técnica exigiu abordagens qualitativa e quantitativa.

A referida técnica é útil quando faltam evidências empíricas, limitadas ou contraditórias, baseando-se na premissa de que uma avaliação precisa e confiável pode ser melhor alcançada consultando um painel de especialistas e aceitando o consenso do grupo. $O$ método Delphi envolve seis etapas: 1) identificação de um problema de pesquisa; 2) conclusão de uma pesquisa na literatura; 3) elaboração de um questionário de declarações; 4) realização de roteiros interativos anônimos ou rodas de questionários; 5) fornecimento de feedback individual e/ou em grupo entre rodadas; e 6) resumindo os resultados. Este processo é repetido até que o melhor nível possível de consenso seja alcançado ou um número predeterminado de rodadas seja completado. Os participantes nunca se encontram ou interagem diretamente. . É uma maneira de estruturar um processo de comunicação em grupo, permitindo que este lide com um problema complexo. (Humphrey-Murto, Varpio, Gonsalves, \& Madeira, 2017; Linstone \& Turoff, 2002).

A amostra populacional foi constituída por doze docentes e preceptores médicos vinculados a um curso público de Medicina no nordeste do Brasil. Como requisito básico para a participação, os profissionais deveriam ser de especialidades médicas que possuíssem área de atuação em cuidados paliativos, conforme definido pela Resolução № 1.973 do Conselho Federal de Medicina (2011). As oito especialidades convidadas foram: clínica médica, medicina intensiva, geriatria, oncologia, cirurgia de cabeça e pescoço, medicina de família e comunidade, pediatria e anestesiologia. 
O convite para participar da pesquisa foi feito através de contato telefônico e, posteriormente, presencial para entrega do Termo de Consentimento Livre e Esclarecido (TCLE).

Os primeiros questionários foram enviados através do correio eletrônico (e-mail). Visto que o envio das respostas não estava acontecendo no tempo esperado, a estratégia de envio foi alterada e passou-se a utilizar o aplicativo de mensagens WhatsApp com um questionário feito no Formulários Google.

Na primeira etapa o questionário contava com questões abertas e se dividia em duas partes. $\mathrm{Na}$ parte I as perguntas serviram para conhecer o perfil profissional do participante: 1. Qual seu nome e data de nascimento?; 2. Há quantos anos exerce a medicina?; 3. Qual sua especialidade?; e 4. Qual o grau de titulação?

A parte II versava sobre experiência pessoal com os cuidados paliativos, bem como sobre o conhecimento e a importância para a formação médica: 1. Você já desenvolveu ou desenvolve práticas de cuidados paliativos na sua rotina de trabalho?; 2. Você identifica no currículo do curso de medicina da Universidade Federal de Alagoas (UFAL) conteúdos ou práticas de cuidados paliativos? Se sim, em qual(ais)?; 3. Quais os conteúdos e práticas em cuidados paliativos que você considera importante para a formação médica em Alagoas? ; 4. Quais as competências em cuidados paliativos você considera necessárias para a formação do médico generalista?

Com as respostas da primeira rodada de perguntas foi possível elaborar uma lista com as competências citadas pelos participantes. Fez-se necessário adaptar algumas palavras em frases dentro do contexto de competências e retirar as sugestões duplicadas, resultando numa lista com 25 opções.

Neste estudo, a definição de competência foi orientada sob a ótica das Diretrizes Curriculares Nacionais (DCN), conforme Resolução CNE/CES n 3 (2014), as quais descrevem que a articulação de conhecimentos, habilidades e atitudes requeridos para o exercício profissional objetivam desenvolver uma prática competente pautada nos princípios da atenção à saúde, gestão à saúde e educação em saúde, proporcionando a capacidade do médico mobilizar diferentes recursos para solucionar com sucesso os problemas da prática profissional. 
$\mathrm{Na}$ segunda etapa houve desinteresse de um participante, que não respondeu em tempo esperado. Logo, 11 participantes se comprometeram com a pesquisa até o final.

Estudos indicam que um número ótimo de participantes na técnica Delphi não deve ser inferior a 10, devido ao comprometimento da relevância das informações, não devendo ultrapassar um total de 30, considerando o maior grau de complexidade (Marques \& Freitas, 2018). Nesta fase da pesquisa foram elaboradas, sem ordem de prioridade, duas listas com 25 temas elencados na primeira etapa pelos participantes da pesquisa.

Nessa lista foram inclusos ainda 15 conteúdos não citados pelos participantes, mas considerados importantes na formação médica, estando presentes no Libro Blanco (Agência Nacional de Avaliação de Qualidade e Credenciamento [ANECA], 2005) - produto da Agência Nacional de Avaliação de Qualidade e Credenciamento (ANECA), que é membro do Comitê Executivo da Associação Européia de Garantia da Qualidade no Ensino Superior (ENQA).

O questionário tinha forma de checklist, possibilitando aos participantes a marcação de quantos itens desejassem em cada lista; o instrumento também contava com uma alternativa que negava todas as sugestões. Uma lista apresentava temas relacionados ao Conhecimento sobre cuidados paliativos, e outra com temas relacionados às Habilidades.

O alto número de itens dificulta a realização da escala Likert, comum no estudo Delphi, mas possibilita elaborar matriz melhor detalhada.

A terceira rodada só foi possível após elaboração da matriz de competências para que os painelistas validassem e fizessem alguma ressalva, caso achassem necessário.

Para elaborar a matriz foi necessário encontrar o consenso entre os participantes da pesquisa sobre os temas elencados na segunda rodada. Foram definidas como competências apenas aquelas escolhidas por mais de dois terços dos painelistas (Marques \& Freitas, 2018; Humphrey-Murto et al., 2017).

O estudo foi submetido e aprovado no Comitê de ética em Pesquisa (CEP/UFAL) com o parecer número 2.769.217. Todos os participantes assinaram o Termo de Consentimento Livre e Esclarecido (TCLE); para preservar o sigilo dos entrevistados, foi atribuída codificação correspondente a uma letra do alfabeto. 


\section{RESULTADOS E DISCUSSÕES}

De uma amostra esperada de 16 participantes, 02 de cada especialidade, na primeira etapa houve adesão de somente 12 especialistas: 02 de clínica médica, 02 geriatras, 01 oncologista, 01 especialista em cirurgia de cabeça e pescoço, 02 médicos de família, 02 pediatras e 02 anestesiologistas. Destes, 10 desenvolvem ou já desenvolveram práticas de cuidados paliativos em sua rotina de trabalho.

Sobre identificar no currículo de Medicina da UFAL alguns conteúdos e práticas, apenas 5 dos 12 participantes citaram alguns momentos: em uma aula no internato, no estágio na Unidade de Terapia Intensiva (UTI) com discussões esporádicas, dentro do módulo de oncologia e hematologia, disciplina eletiva de cuidados paliativos e na enfermaria de cuidados paliativos no estágio de clínica médica.

A primeira rodada do questionário resultou em vinte e cinco sugestões de competências, às quais foram adicionadas outras quinze presentes no Libro Blanco (ANECA, 2005). Para a segunda rodada (Kang et al., 2013), utilizou-se a técnica Delphi, de modo semelhante, para desenvolver competências em cuidados paliativos: na primeira rodada foi apresentado um checklist com sugestões de competências; na segunda foram acrescentadas sugestões dos participantes na rodada anterior.

$\mathrm{Na}$ segunda etapa do estudo, os participantes identificaram a maioria das competências como necessárias para a formação médica (Tabela 1).

Das 40 competências sugeridas, apenas 10 não foram consenso entre os painelistas (menos de $70 \%$ de citação): saber particularidades do cuidado paliativo na pediatria; conhecer o papel da fisioterapia, principalmente pós-operatória; conhecer o tratamento das patologias dos pacientes em cuidados paliativos; saber montar uma equipe de cuidados paliativos com suporte à família e aos profissionais; manejar o estresse da equipe de saúde; realizar cuidados com a boca do paciente; manejo geral das reações transfusionais; realizar outras técnicas analgésicas; realizar Infiltração de anestésicos e /ou esteroides; e realizar bloqueio neural. 
Tabela 1. Temas selecionados (\%) para compor a matriz de competências em cuidados paliativos na graduação médica.

\section{Conhecimentos}

- Conhecer o conceito de cuidados paliativos.

- Conhecer o critério de indicação para cuidados Paliativos.

- Conhecer sobre cuidados paliativos na Atenção Básica.

- Conhecer a atitude do médico diante da morte de seu paciente.

- Conhecer o conceito de doença terminal

- Compreender a relação da bioética com os cuidados paliativos.

- Saber encaminhar para o internamento quando necessário.

- Cuidados com a boca, mucosites, halitoses e infecções oral.

- Conhecer o conceito de dor total.

- Saber sobre a farmacologia e uso clínico de opioides em cuidados paliativos.

- Reconhecer aspectos psicológicos, sociais e espirituais na abordagem de cuidados

paliativos com o paciente e seu familiar.

- Conhecer aspectos básicos da Atenção domiciliar.

- Identificação do luto patológico.

- Entender sobre alimentação em cuidados paliativos.

- Saber Particularidades do cuidado paliativo na pediatria.

- Conhecer o papel da fisioterapia, principalmente pós-operatória.

- Conhecer o tratamento das patologias dos pacientes em cuidados paliativos.

Habilidades

- Agir com paciência em cuidados paliativos.

- Exercer a empatia.

- Controlar a dor e outros sintomas mais comuns.

- Comunicar-se adequadamente, incluindo a comunicação de má notícia.

- Trabalhar em Equipe Multidisciplinar/Interdisciplinar.

- Fazer a evolução da dor com uso de escalas.

- Apresentar disponibilidade para atuar em cuidados paliativos.

- Ter uma visão generalista.

- Aplicar medicina baseada em evidência.

- Fazer história clínica do paciente com doença terminal.

- Fazer evolução médica dos paciente com doenças terminal.

- Manejar o paciente em seus últimos dias de vida no domicílio ou no hospital.

- Construir um projeto terapêutico singular.

- Dominar situações de urgência em paciente oncológico terminal

- Prescrever hemoderivados.

- Articular com a rede de atenção à saúde o atendimento secundário.

- Saber montar uma equipe de cuidados paliativos com suporte à família e aos profissionais.

- Manejar o estresse da equipe de saúde.

- Realizar cuidados com a boca do paciente.

- Manejo geral das reações transfusionais.

- Realizar outras técnicas analgésicas

- Realizar Infiltração de anestésicos e /ou esteroides.

- Realizar bloqueio neural.

Houve algumas discordâncias destes resultados com a literatura. Das 15 competências acrescentadas do Libro Blanco, 6 não foram consenso entre os painelistas: realizar bloqueio neural, conhecer o papel da fisioterapia, realizar cuidados com a boca, infiltração de 
analgésicos, manejo das reações transfusionais e o manejo do estresse na equipe de saúde. Magaña, Santamaría, Casanovas e López (2018), em estudo sobre universidades de El Salvador, recomendam o treinamento de novas equipes para o manejo de estresse, especialmente em cuidados paliativos.

Chenge e Teh (2014), em estudo na Austrália, recomendam trabalhar em cuidados paliativos com equipes eficazes e com profissionais capazes de exercitar 0 autoconhecimento, conscientes das forças pessoais e fragilidades, atentos ao Burnout (EAPC, 2013).

Desta forma, infere-se a importância da capacidade de gerenciamento do estresse por meio de equipe para auxiliar no controle dos sintomas dos pacientes e seus familiares, e do tema para a formação de profissionais de saúde.

Apesar deste estudo não avaliar a importância dada pelos painelistas a cada competência, como em outros estudos, foi possível observar uma abrangência a vários aspectos dos cuidados paliativos.

Em estudo realizado na Coreia (Kang et al., 2013), as competências médicas de mais importância foram semelhantes às mais votadas neste estudo: controle de sintomas em pacientes em cuidados paliativos e os cuidados no final de vida com o paciente e seu familiar.

Cuidados paliativos na pediatria não foi considerada competência necessária para o médico generalista neste estudo e, de forma semelhante, não teve importância como competência médica no estudo realizado na Coreia, sendo considerada competência para a enfermagem (Kang et al., 2013).

Uma matriz de competência é um instrumento importante na formação médica, visto que expressa o consenso coletivo e o conteúdo necessário a todo médico generalista, não necessariamente presente em uma disciplina específica ou no docente (Marques \& Freitas, 2018).

A partir da análise das respostas da segunda rodada, foi possível elaborar a matriz de competência com as respostas que foram consenso (Quadro 1). 
Quadro 1. Matriz de competências elaborada com as respostas que foram consenso (> 70\%) entre os participantes

\begin{tabular}{|c|c|}
\hline Conhecimentos & Habilidades \\
\hline $\begin{array}{l}\text { - Conhecer o conceito de cuidados paliativos. } \\
\text { - Conhecer o critério de indicação para cuidados } \\
\text { Paliativos. } \\
\text { - Conhecer sobre cuidados paliativos na Atenção } \\
\text { Básica. } \\
\text { - Conhecer a atitude do médico diante da morte de } \\
\text { seu } \\
\text { paciente. } \\
\text { - Conhecer o conceito de dor total. } \\
\text { - Conhecer o conceito de doença terminal } \\
\text { - Conhecer os aspectos básicos da atenção } \\
\text { domiciliar, } \\
\text { - Compreender a relação da bioética com os } \\
\text { cuidados } \\
\text { paliativos. } \\
\text { - Saber sobre a farmacologia e uso clínico de } \\
\text { opioides } \\
\text { em cuidados paliativos. } \\
\text { - Cuidados com a boca, mucosites, halitoses e } \\
\text { infecções } \\
\text { orais. } \\
\text { - Entender sobre alimentação em cuidados } \\
\text { paliativos. } \\
\text { - Saber encaminhar para o internamento quando } \\
\text { necessário. } \\
\text { - Reconhecer aspectos psicológicos, sociais e } \\
\text { espirituais } \\
\text { na abordagem de cuidados paliativos com o } \\
\text { paciente } \\
\text { e seu familiar. } \\
\text { - Identificação do luto patológico. }\end{array}$ & $\begin{array}{l}\text { - Exercer a empatia. } \\
\text { - Agir com paciência em cuidados paliativos. } \\
\text { - Trabalhar em Equipe Multidisciplinar } \\
\text { /Interdisciplinar } \\
\text { - Ter uma visão generalista. } \\
\text { - Aplicar medicina baseada em evidência. } \\
\text { - Fazer história clínica do paciente com } \\
\text { doença terminal. } \\
\text { - Controlar a dor e outros sintomas mais } \\
\text { comuns. } \\
\text { - Articular com a rede de atenção à saúde o } \\
\text { atendimento secundário. } \\
\text { - Apresentar disponibilidade para atuar em } \\
\text { cuidados paliativos. } \\
\text { - Dominar situações de urgência em paciente } \\
\text { oncológico terminal. } \\
\text { - Prescrever hemoderivados. } \\
\text { - Fazer evolução médica dos pacientes com } \\
\text { doenças terminais. } \\
\text { - Fazer evolução da dor com uso de escalas. } \\
\text { - Comunicar-se adequadamente, incluindo a } \\
\text { comunicação de má notícia. } \\
\text { - Manejar o paciente em seus últimos dias de } \\
\text { vida no domicílio ou no hospital. } \\
\text { - Construir um projeto terapêutico singular. }\end{array}$ \\
\hline
\end{tabular}

Neste estudo, a definição de competência esteve orientada pela ótica das DCN, conforme Resolução CNE/CES no 3 (2014), as quais articulam conhecimentos, habilidades e atitudes para a formação médica e têm por objetivo desenvolver uma prática profissional competente em três áreas: I - Atenção à saúde, II - Gestão à saúde e III - Educação em saúde, proporcionando ao médico condições para mobilizar diferentes recursos na resolução dos problemas do cotidiano profissional com sucesso.

Observa-se que a maior quantidade de competências (vinte e sete) elencadas pelos participantes está relacionada com a atenção à saúde. As competências relacionadas com a Gestão em Saúde foram: Trabalhar em Equipe Multidisciplinar/Interdisciplinar e Articular com a rede de atenção à saúde o atendimento secundário. Segundo a Resolução CNE/CES no 3 (2014), dentro da área de Gestão em Saúde, para a formação médica é necessário 
compreender a importância do trabalho colaborativo em equipes de saúde, respeitando normas institucionais dos ambientes de trabalho e agindo com compromisso éticoprofissional, superando a fragmentação do processo de trabalho em saúde. Além disso, favorecer a articulação de ações de profissionais e serviços, apoiando a implantação de dispositivos e ferramentas que promovam a organização de sistemas integrados de saúde.

Apenas uma competência foi relacionada à Educação em Saúde: aplicar medicina baseada em evidência, compatível com o comportamento de valorização da pesquisa científica a favor da assistência à saúde. Entretanto, a área de Educação em Saúde poderia ser melhor representada neste estudo, caso identificada alguma competência mais especificamente voltada à área da pesquisa e promoção à saúde. Está explícita nas DCN a necessidade do graduando ser corresponsável pela sua formação, o que inclui favorecer o desenvolvimento científico e tecnológico voltado para a atenção das necessidades de saúde individuais e coletivas, por meio da disseminação das melhores práticas e do apoio à realização de pesquisas de interesse da sociedade. Outrossim, identificar a necessidade de produção de novos conhecimentos em saúde, a partir do diálogo com a própria prática, a produção científica e o desenvolvimento tecnológico disponíveis. Semelhante a este estudo, na pesquisa de Kang et al. (2013), os domínios educação e pesquisa tiveram menos importância que os demais na avaliação.

Nesta pesquisa foi possível identificar que as recomendações mais votadas pelos painelistas foram: conhecimento sobre cuidados paliativos, incluindo critérios de indicação; cuidados paliativos na atenção básica; controle de sintomas; trabalho multiprofissional e interprofissional; habilidades de comunicação e bioética. Semelhante a este estudo, também é possível verificar em outras pesquisas como principais recomendações: conhecimento sobre cuidados paliativos, controle da dor e outros sintomas, habilidade de comunicação e ética (Lehto, Hakkarainen, Kellokumpu, Pirkko, \& Saarto, 2017; Fitzpatrick, Heah, Patten, \& Ward, 2017; Nakamura et al., 2017) recomendam os assuntos acima citados como principais e que estejam presentes na medicina clínica desenvolvendo um currículo integrado. Além destas principais recomendações, Mutto et al. (2005) acrescentam a importância do apoio psicossocial aos pacientes e familiares.

Alguns estudos consideram a ética médica como uma das competências mais importantes. Horowitz et al. (2014) afirmam que a Associação das Faculdades Americanas recomenda a presença do conteúdo sobre consentimento informado e diretrizes antecipadas de vontade 
no currículo para formação médica. Chenge e Teh (2014) não especificam os conteúdos específicos à ética, mas enfatizam a importância deste domínio no início do curso de Medicina; Suvarnabhumi et al. (2013) e Lehto et al. (2017) orientam de acordo com a recomendação da Associação Europeia de Cuidados paliativos relacionando ética e direito.

Um novo desafio se impõe após a elaboração da matriz de competências: onde, quando e como esta matriz poderá ser incorporada ao currículo médico? Esse é um desafio que deve considerar a contextualização epidemiológica e cultural da região onde o curso está implantado, além de estratégias inovadoras de gestão do ensino que considerem o SUS como referência de atenção, trabalho e formação em saúde.

\section{CONCLUSÕES}

A técnica Delphi é uma importante ferramenta de pesquisa qualitativa que objetiva o consenso de um grupo de especialistas a respeito de um determinado tema complexo e abrangente. Possibilita opiniões bem refletidas, pensamentos independentes, que convergem em uma melhor tomada de decisão para a elaboração de um cenário futuro e para transformação de uma realidade.

Diante do número crescente de pacientes em cuidados paliativos, reconhece-se a necessidade de ampliar a formação nesta área. Elaborar e implementar uma matriz de competência em cuidados paliativos é uma estratégia para garantir a reflexão e a vivência discente, sistemática, desde o início do curso.

A técnica Delphi proporcionou a elaboração de uma matriz de competências em cuidados paliativos de acordo com as Diretrizes Curriculares Nacionais para o curso de Medicina, com a colaboração de médicos de diversas especialidades. Esta ferramenta pode ser considerada útil para o diagnóstico situacional, planejamento de ações e para auxílio à gestão institucional do ensino superior em saúde. Deve-se considerar, no entanto, o rigor científico para evitar possíveis vieses de seleção no uso da técnica.

O estudo possibilitou o desenvolvimento de uma matriz de competências em cuidados paliativos, necessária para a formação médica e suficiente para garantir a atenção humanizada à saúde de parcela significativa da população. 


\section{REFERÊNCIAS}

Agência Nacional de Avaliação de Qualidade e Credenciamento. (2005). Libro Blanco: Título de médico. Granada, Espanha: Agência Nacional de Avaliação de Qualidade e Credenciamento.

Associação Europeia de Cuidados Paliativos (EAPC). (2013). Competências centrais em cuidados paliativos: Um guia orientador da EAPC sobre educação em cuidados paliativos: parte 1. Jornal Europeu de Cuidados Paliativos, 20(2), 86-91.

Carvalho, J. A. M., \& Garcia, R. A. (2003). O envelhecimento da população brasileira: Um enfoque demográfico. Cadernos de saúde pública, 19(3), 725-733. doi: 10.1590/S0102-311X2003000300005

Cheng, R., \& Teh, A. (2014). Palliative care in Australian medical student education. Medical Teacher, 36(1), 8283. doi: 10.3109/0142159X.2013.818630

Council of Europe. (2003). Recommendation Rec (2003) 24 of the Committee of Ministers to member states on the organisation of palliative care. Spain, Europe: Council of Europe. Retrieved from http://www.coe.int/t/dg3/health/Source/Rec(2003)24_en.pdf.

Denney-Koelsch, E. M., Horowitz, R., Quill, T., \& Baldwin, C. D. (2018). An integrated, developmental fouryear medical school curriculum in palliativecare: A longitudinal content evaluation based on national competency standards. Journal of Palliative Medicine, 1(9), 1221-1233. doi: 10.1089/jpm.2017.0371

Fitzpatrick, D., Heah, R., Patten, S., \& Ward, H. (2017). Palliative care in undergraduate medical education - How far have we come? American Journal of Hospice and Palliative Medicine, 34(8), 762-773. doi: $10.1177 / 1049909116659737$

Floriani, C. A., \& Schramm, F. R. (2007). Desafios morais e operacionais na inclusão dos cuidados Paliativos na rede de atenção básica. Cardernos de saúde pública, 23(9), 2072-2080. doi: 10.1590/S0102$311 \times 2007000900015$

Horowitz, R., Gramling, R., \& Quill, T. (2014) Palliative care education in US medical schools. Medical Education, 48(1), 59-66. doi: 10.1111/medu.12292

Humphrey-Murto, S., Varpio, L., Gonsalves, C., \& Madeira, T. J. (2017). The use of the Delphi and other consensus group methods in medical education research: A review. Academic Medicine, 92(10), 14911498. doi: $10.1097 /$ ACM.0000000000001812

Kang, J., Kim, Y., Yoo, Y. S., Choi, J. Y., Koh, S. J., Jho, H. J. ... Jones, D. (2013). Developing competencies for multidisciplinary hospice and palliative care professionals in Corea. Supportive Care in Cancer, 21(10), 2707-2717. doi: 10.1007/s00520-013-1850-3

Lehto, J. T., Hakkarainen, K., Kellokumpu, L., Pirkko, L., \& Saarto, T. (2017). Undergraduate curriculum in palliative medicine at Tampere University increases students' knowledge. BMC Palliative Care, 16(13). doi: 10.1186/s12904-016-0182-8

Linstone, H. A., \& Turoff, M. (2002). The Delphi method: Techniques and applications. New Jersey Institute of Technology. Disponível em: https://web.njit.edu/ turoff/pubs/delphibook/index.html

Magaña, M. A. F., Santamaría, K. P., Casanovas, J. G., \& López, M. (2019). Teaching of palliative care in medical schools in El Salvador. Educación Médica, 20(1), 143-147. doi: 10.1016/j.edumed.2017.09.010

Marques, J. B. V., \& Freitas, D. (2018). Método Delphi: Caracterização e potencialidades na pesquisa em Educação. Revista Pro-Posições, 29(2), 389-415.

Ministério da Saúde (2007). Integração de informações dos registros de câncer brasileiros. Revista saúde pública, 41(5), 865-868. 
Mutto, E. M., Bunge, S., Vignaroli, E., Bertolino, M., Villar M. J., \& Wenk, R. (2014). Medical students' palliative care education in a latin american university: A three-year experience at austral university in Buenos Aires, Argentina. Journal of palliative medicine, 17(10), 1137-1142.

Nakamura, Y., Takamiya, Y., Saito, M., Kuroko, K., Shiratsuchi, T., Oshima, K., ... Miyake, S. (2017). A survey of palliative medicine education in Japan's undergraduate medical curriculum. BMC Palliative Care, 16(1).

Oliveira, J. R., Ferreira, A. C., \& Rezende, N. A. (2013). Ensino de bioética e cuidados paliativos nas escolas médicas do Brasil. Revista Brasileira de Educação Médica, 37(2), 285-290. Disponível em http://www.scielo.br/pdf/rbem/v37n2/17.pdf

Resolução CNE/CES no 3. (2014, Junho). Institui as diretrizes curriculares nacionais do curso de graduação em medicina e dá outras providências. Brasília, Brasil: Ministério da Educação. Disponível em: http://portal.mec.gov.br/index.php?option=com_docman\&view=download\&alias=15874-rces00314\&category_slug=junho-2014-pdf

Resolução no 41. (2018, Outubro). Dispõe sobre as diretrizes para a organização dos cuidados paliativos, à luz dos cuidados continuados integrados, no âmbito Sistema Único de Saúde (SUS). Brasília, Brasil: Ministério da Saúde. Disponível em: https://portalarquivos2.saude.gov.br/images/pdf/2018/novembro/23/RESOLUCAO-N41.pdf

Resolução CFM no 1.973 (2011, Agosto). Dispõe sobre a nova redação do Anexo II da Resolução CFM no 1.845/08, que celebra o convênio de reconhecimento de especialidades médicas firmado entre o Conselho Federal de Medicina (CFM), a Associação Médica Brasileira (AMB) e a Comissão Nacional de Residência Médica (CNRM). Brasília, Brasil: Conselho Federal de Medicina.

Souza, M. F. M., Malta, D. C., França, E. F., \& Barreto, M. L. (2018). Transição da saúde e da doença no Brasil e nas Unidades Federadas durante os 30 anos do Sistema Único de Saúde. Ciência \& Saúde Coletiva, 23(6), 1737-1750. doi: 10.1590/1413-81232018236.04822018

Suvarnabhumi, K., Sowanna, N., Jiraniramai, S., Jaturapatporn, D., Kanitsap, N., Soorapanth, C., ... Phungrassami, T. (2013) Situational analysis of palliative care education in Thai medical Schools. Palliative Care: Research and Treatment, 7, 25-29. doi: 10.4137/PCRT.S12532

World Health Organization. (2002). National cancer control programmes: Policies and managerial guidelines. 2 ed. Geneva, Switzerland: World Health Organization. 